\title{
Resources Indexation-Based E-Learning System
}

\author{
${ }^{1}$ Ibtissam Azzi, ${ }^{2}$ Abdelhay Radouane, ${ }^{3}$ Adil Jeghal, ${ }^{4}$ Hamid Tairi \\ ${ }^{1}$ Laboratoire LIIAN, Faculté des Sciences Dhar Mehraz, U.S.M.B.A. Fès, Maroc \\ ${ }^{2}$ Département d'informatique, Centre Régional des métiers de l'éducation et de Formation, C.R.M.E.F. \\ Fès, Maroc, 3Laboratoire LIIAN, Faculté des Sciences Dhar Mehraz, U.S.M.B.A. Fès, Maroc \\ ${ }^{4}$ Laboratoire LIIAN, Faculté des Sciences Dhar Mehraz, U.S.M.B.A. Fès, Maroc

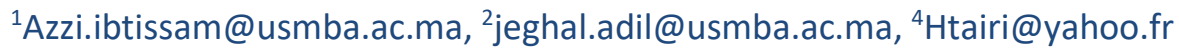

\begin{abstract}
We propose in this article a study that illustrates the techniques used to represent the educational objects which serve to facilitate their reuse. We will enrich this study by taking into account the semantics of the contents of the learning objects while including the metadata as parameters of indexing. Another contribution of our study concerns the fact that this indexation relies on ontologies which allow a better semantic representation and facilitate communication between the machine and the users. On the other hand, we will discuss the current standards of indexing while discussing the cases of their use to try to extend the use of these standards in other situations requiring the indexing of resources in $E$-Learning systems.
\end{abstract}

Keywords- Resources indexation; Metadata; Ontology; Standards; E Learning.

\section{Introduction}

The deployment of information and communication technologies has taken a major boom in the professional and personal domains of individuals, and computing has become a necessary and powerful medium for improvement and innovation. Indeed, with the era of the Internet, the field of education and training has found a room to optimization with e-learning which relies on the provision of learning content through learning designs in a digital environment. However, with the exponential growth in the number of available resources, it is increasingly difficult for learners and teachers to find the learning content that best meets their needs. To enable localization, access, sharing and re-use of these resources, it is essential to describe them. One solution may come from the use of information derived from the descriptions of learning resources. On the other hand, besides content development, the creation of such resources poses many difficulties for its designers. The use of these resources in learning designs implies not only that the resource be divided into coherent learning objects but also the activities associated with the pedagogical objectives be defined. Existing resources are difficult to re-use because they have not been designed for this purpose; they often correspond to simple on-line presentations of documents that have not been created specifically for their use in learning environments. [1] finds insufficient or no application of a pedagogical approach, whether in the presentation of learning resources or the sequencing of learning activities in current tools. On the other hand, to be reusable, a resource must be indexed so as to know at the same time the content, the educational objectives aimed at, the conditions of reusability, etc. In the literature one finds standards that offer solutions for the problem of indexing in the framework 
Ibtissam Azzi, Abdelhay Radouane, Adil Jeghal, Hamid Tairi; Resources Indexation-Based E-Learning System, Transactions on Machine Learning and Artificial Intelligence, Vol 5 No 4, August, (2017); pp: 1-7

of E- Learning. We will discuss their advantages and limitations in this article which is structured in the following way: in section II we will clarify the value of introducing resource indexing in the solution of problems related mainly to the personalization of learning in systems of $E$-Learning. Subsequently, in Section III, we will discuss the role of metadata in the semantic description of the resources while giving an overview on the different standards using the metadata. We will also discuss in section IV the notion of ontologies where we will discuss the various difficulties encountered in their conception. Section V describes the reuse of the ontologies. On the contrary in the conclusion, we will present our point of view concerning the exploitation of ontologies in the indexation of resources in particular, we will exploit the important property of ontologies namely the property of integration which allows the development of ontologies already designed to create another. We want to create an ontology that takes into account the learner's learning style in order to personalize it. This will be a promising prospect in our future work.

\section{Resource Indexation In E Learning Systems}

The definition of a learning object has been the subject of several debates. According to the Learning Object Metadata (LOM) standard [2], a learning object is defined as any numeric or non-numerical entity that can be used, reused or referenced during learning activities. This definition is considered by [3] to be very broad because it may include an object, a person or an idea. [4] refined this definition by adding that a learning object is a unit of learning content that is independent, autonomous and predisposed to be reused in multiple pedagogical contexts. [5] emphasizes that the notion of learning object remains unclear, because some definitions are either too broad or too narrow in relation to the use that can be made of them. In order to remedy this problem of definition we consider that a learning object is a material that can be selected, combined with another material according to the needs of the teachers and the learners. It is also a learning content which must exist as such, and which can be easily searched and indexed [6]. To enable indexing and retrieval of these learning objects, it is necessary to use a set of metadata. The fundamental idea behind the creation of learning objects is the possibility of building even small components or elements that can be reused several times in different learning contexts. [7] argues that, often when teachers or creators of learning content first access learning materials, they decompose it into its constituent elements and then assemble them in order to construct one which supports their pedagogical objectives. If teachers or content creators could have these resources as small units, this first stage of decomposition would be bypassed. This can greatly increase the speed and effectiveness of creating new resources [3]. However, in order for these small units to be reused they must be able to find them. In order to facilitate the adoption of the "learning object" approach, the IEEE 2 consortium created the Learning Technology Standards Committee (LTSC) in 1996 to develop and promote standards for learning technology. The committee then chose the term learning object to describe these small reusable units. The indexing of a resource makes it possible to describe it for better use because a non-indexed resource is an unexploited resource which is impossible to find.

\section{Metadata And Lom, Scorm Standards}

Metadata is semantic data that describes resources. Tim Berners Lee, the inventor of the web, said that digital information resources must be understandable by human beings and processable by software. The indexation must then be precise, which sometimes implies a very large number of fields to fill, more than fifty in the LOM. Some of the indexing can be done automatically, such as automatic recognition of the 
Transactions on Machine Learning and Artificial Intelligence Vol 5 No 4, Aug 2017

file format by the software. But the description of the pedagogical objective of a resource, for example, should be done manually.

Next, the display of the metadata fields will be adapted according to the type of user: a user will not need to see the same fields as an indexer. On the web, search engines like Google in principle make it possible to find all that is accessible. This shows that metadata is really needed. They help not only to find resources more easily, but also to evaluate them, even if they are not the same descriptors that are used in both cases. Finally, metadata also facilitates maintenance, through the management of different versions of a document, and automatic assembly of resources. It is then essential to anticipate and think about all the uses of a resource before defining the set of associated metadata. On the other hand, indexing is complex and requires some standardization. Indeed, in certain contexts, it is possible to impose the indexing system, the descriptors, the software, the file formats, the operating system, the instantiation of the Learning Object Metadata (LOM) standard. However, a change in context will then encounter some problems and involve transforming data. In other cases, the problem is rather how to interact with other platforms. The aim is therefore not to repeat the indexing each time a change of platform or interlocutors occurs. The use of standards or standards is then made mandatory. Indeed, standards are the only means that allow interoperability and evolution of systems over time. A norm or a standard can not necessarily meet everyone's needs, but must be scalable. Norms and standards must be built on a basis of common needs. Similarly, for indexing to survive software and hardware, it must imperatively be based on standards. Transcripts allowing the passage from one environment to another will necessarily be written when norms or standards evolve. Standards and norms therefore make it possible to maintain resources and, thanks to indexation, they can be guaranteed accessibility and interoperability through exchanges between systems. Resources can then be reused and adapted.

The current e-Learning standards offer a partial solution for reusability. In particular, SCORM (Sharable Content Object Reference Model) [8] and LOM (Learning Object Metadata) help to homogenize representations of learning resources and facilitate interoperability. LOM proposes different metadata necessary for the description of the learning resources, but this description is not enough, it lacks the semantic representation of the contents. SCORM for its part, allows the structuring of the contents of the learning resources and their relations with the environment of use. However, the representations obtained via these standards are not sufficient to allow and ensure the reusability of resources or parts of resources because they take into account neither the semantic content of the metadata they introduce nor the relations that exist between each learning object. To remedy these problems, representations of "learning objects" which are based on a representation of knowledge in the form of ontologies have been proposed. They deal with the reusability aspect of these documents, in particular their semantic indexing, the pedagogical aspect which makes it possible to learn the knowledge conveyed in the documents.

\subsection{LOM (Learning Object Metadata)}

At present, the most complete and most suitable standard for the field of education and e-learning remains the LOM.

The LOM or Metadata for Educational Objects standard is a standard developed in 2002 by the IEEE consortium, which defines the structure of a metadata instance for an educational object. It consists of nine categories (general, life cycle, meta-metadata, educational, technical, rights, relation, annotation, classification) each performing a different function. The descriptors of the LOM can be used in the design 
Ibtissam Azzi, Abdelhay Radouane, Adil Jeghal, Hamid Tairi; Resources Indexation-Based E-Learning System, Transactions on Machine Learning and Artificial Intelligence, Vol 5 No 4, August, (2017); pp: 1-7

of E-learning systems for indexing learning objects. For this they must be implemented in a structured language. The representation of the abstract model in a specific format is called "binding".

For LOM metadata, there are two metadata: XML binding and RDF binding. The use of a language such as XML (Extensible Markup Language) makes it possible to perceive the structure of the LOM and facilitates the exchange of metadata between different systems. If the XML binding of the LOM has the merit of being easy to implement, it remains insufficient for the representation of the elements of the LOM since it does not make it possible to express the semantics of these elements.

While XML structures metadata, RDF (Resource Description Framework), poses on XML a framework for defining these metadata. This is why RDF is referred to as metalanguage metadata. It is intended to structure the information accessible on the web and to index it effectively. However, whether they are implemented in XML or RDF, using LOM metadata poses a number of problems. Indeed, according to [9] to manage resources with the metadata one can enumerate many problems encountered when using the metadata of the LOM (complexity of the structure, important number of elements, difficulty to inform some fields, etc.).

\subsection{SCORM (Sharable Content Object Reference Model)}

It is a model for the assembly of web contents and a learning environment for learning objects. Its aim is to put in place the right structure of the content of the course and its interactions with its environment. The structuring of the content of the teaching modules according to the SCORM model makes it possible to reuse them in other modules for different training or systems. Moreover, it improves the dialogue between the learning objects and the system on the one hand, and between the actors and the system on the other.

SCORM deals with several elements, for example:

- $\quad$ Packaging: its objective is the transmission of content from one platform to another, the import or export of contents learning objects to make them available to others. It is equally interested in the structuring of learning objects.

- Meta data: they are from LOM and aim to share standard information that describes the nature and purpose of the content.

- Communication or execution environment: determines the communication with a web environment.

- $\quad$ Sequencing and navigation: defines a method of representing navigation between learning objects.

\section{Ontologies}

In order to solve some of the problems associated with lack of semantics, ontologies have been introduced [10], [11], [12] and [13]. According to [14], an ontology groups the concepts that represent the whole knowledge of a domain into an explicit and formal specification. It shows the relations as well as the association rules that exist between these concepts in order to allow, on the one hand, the computer the production of new knowledge by means of an inference and, on the other hand, to allow the man and the computer to give common meanings to the terms used in a field of activity in order to remove any ambiguity during the treatments. 
An ontology is translated by the following elements:

- $\quad$ Concepts: also called terms or classes of ontology, correspond to the relevant abstractions of a segment of reality (the domain of the problem), selected according to the objectives that are set and the application envisaged for the " ontology.

- Relationships: A relationship is a link between concepts and describes a type of interaction between concepts.

- The axioms: constitute assertions, accepted as true, about the abstractions of the domain translated by the ontology.

- The instances: constitute the extensional definition of ontology; these objects convey knowledge (static, factual) about the domain of the problem.

However, the design of an ontology is a matter of ontological engineering [15]. In the literature, we can find several methodologies for designing ontologies dedicated to E -Learning [16], [17], [18], [19] and [20]. Reference [21] proposes an ontology of courses that breaks down into 3 sub-parts describing the content, context and structure. The content is commonly referred to as the ontology of the domain. The other two parts are related to the pedagogical aspect (structuring in chapters, nature of the parts, etc.). Reference [22] considers that domain and pedagogy can be combined within a single ontology. The ontology provides a set of Concepts and relationships modeling a domain, a pedagogical strategy, etc. Therefore this requires a modeling language. One can cite the UML (Unified Modeling Language). One of the advantages of the UML schema compared to other formalisms is that it offers a direct connection to the world of software engineering. In addition, there are already tools to manipulate the models. From these diagrams are derived a vocabulary and a metadata schema for indexing resources in XML with respect to this model The other favours which ontologies offer lies in their property of integration. Indeed, one can use ontologies already conceived to conceive a new ontology adapted to our needs.

\section{Reuse of ontologies}

In this context, work highlighted the possibility of re-use of certain ontologies. Ontologies of field and task, nonspecific to a given application, can be regarded as reusable [23], in particular if they specialize the concepts of a high level ontology. In [24], we identified 4 stages which the originator must carry out to use of existing ontologies:

- Specification and formalization of the needs.

- $\quad$ Specification and the formalization of the requirements in terms for operational features for the software.

- Appropriation of ontologies and creation of an ontology of application.

- It is a question of re-using an existing ontology of field or an existing ontology of task and then to create an ontology of application by using these two ontologies.

- Operationnalisation.

- The operationnalisation of ontology thus consists of the computer specification of the operations applicable to the concepts in an operational language.

- $\quad$ Finalization of the establishment and data model.

It is a question of choosing the environment of establishment of the application, with regard to the management of this data and their establishment then the originator/developer establishes his interfaces and its application in a language targets (e.g.:Java). 
It is in this direction that we wish to act in our future work while trying to use ontologies of field and ontologies of pedagogy to create an ontology which gives an account of the preferences of the user in particular his learning style. For that, we will adopt the model which represents knowledge relative to the various aspects of a document and its uses. It is described by the following schema [25] and [26]:

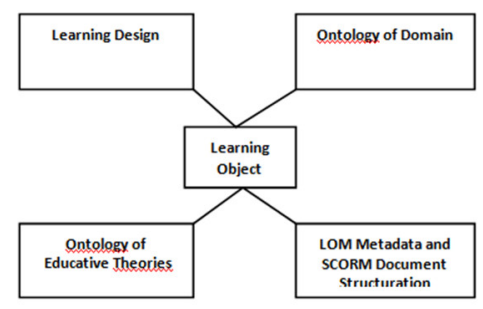

\section{Conclusion}

In this article, we have clarified the value of indexing resources in E -Learning systems and have also given an overview of the problem of indexing in these systems. In addition, we have overflowed the various descriptions existing in the literature capable of indexing the resources. In this sense, we discussed the metadata and their ability to describe the semantics of resources in particular, we have dealt with the LOM and SCORM standards where we have recalled the advantages and limitations of these standards. We have also clarified the need to introduce ontologies as a means of remedying the deficiencies encountered in the description made by the metadata. Despite these different constraints, and because the scope of ontologies is wide, and the problems related to e-learning are diversified, we wish to develop an ontology in our future work, taking into account the learner's learning style. This ontology will be associated with LOM metadata.

\section{REFERENCES}

[1] Psyché V., Bourdeau J., Nkambou R. et Mizoguchi R."Making Learning Design Standards Work with an Ontology of Educational Theories". Artificial. Intelligence in Education (AIED 2005), 725-731.

[2] LOM (2002). LOM standard, document IEEE 1484.12.1-2002.

[3] WILEY D." Connecting learning objects to instructional design theory : A definition, a metaphor, and a taxonomy". The Instructional Use of Learning Objects, p. 571-577, 2002.

[4] POLSANI P."Use and Abuse of Reusable Learning Objects". Journal of Digital Information, 3(4), $2003-02$.

[5] BOURDA Y. "Objets pedagogiques, vous avez dit objets pedagogiques ?" Cahier GUTenberg n 39, 40, 7179, 2001.

[6] ABEL M.-H. "Apport des Memoires Organisationnelles dans un contexte d'apprentissage". Habilitation a diriger des recherches, UTC, 2007.

[7] REIGELUTH C. "A New Paradigm of ISD ? "Educational Technology -SADDLE BROOK NJ-, 36, 13-20, 1996.

[8] SCORM. Le modèle SCORM (2004). http://www.adlnet.org 
Transactions on Machine Learning and Artificial Intelligence Vol 5 No 4, Aug 2017

[9] MICHEL A. "La gestion des ressources avec les métadonnées". Journee Normes et standards educatifs, 26, 2004.

[10] Gruber T." A transaction approach to portable ontology specification ", Knowledge Acquisition, 5(2) p. 199220. 1993

[11] Uschold M., Gruninger M." ONTOLOGIES : Principles, Methodes and Applications", Knowledge Engineering Review. vol. 11, $\mathrm{n}^{\circ} 2,1996$.

[12] Borst W.N."Construction of Engineering Ontologies", PhD Thesis, University of Twente, Enschede, 1997.

[13] Giraldo G., Reynaud C." Construction semi-automatique d'ontologies à partir de DTDs relatives à un même domaine", Actes de IC'2002, Rouen, mai 2002.

[14] Studer R., Benjamins VR. et Fensel D. "Knowledge Engineering: Principles and Methods". Data and Knowledge Engineering (DKE), 25(1-2), p 161-197, 1998.

[15] GÓMEZ-PÉREZ A. "Ontological Engineering". Tutorial ECAI'98, Budapest, Hongrie, août 1998.

[16] Desmoulins C. et Grandbastien M. "Des ontologies pour la conception de manuels de formation à partir de documents techniques". Revue Sciences et Techniques éducatives, 2002.

[17] Duitama J. F., Defude B., Bouzeghoub A., Carpentier C. "A Framework for the generation of Adaptive Courses Based on Semantic Metadata", Proc. MAWIS Workshop, with OOIS'03 Conference, Geneva, September, 2003.

[18] Dehors S., Faron Zucker C., Giboin A., Stromboni J. P. "Un Web Sémantique de Formation par Questionnement". Acte des journnées Extraction et Gestion des Connaissances 2005. EGC 2005

[19] Ranwez S. et Crampes M. "Conceptual Documents and Hypertext Documents are two Different Forms of Virtual Document", Workshop "on Virtual Documents Hypertext Funcionality and the Web" of the 8th World Wide Web Conf., Toronto, 1999.

[20] Iksal S. "Spécification Déclarative et Composition Sémantique pour des Documents Virtuels Personnalisables", Thèse de Doctorat, EHESS, Brest, France, 2002.

[21] STOJANOVIC L., STAAB S. \& STUDER R. "Elearning based on the semantic web", WebNet2001 - World Conference on the WWW and Internet, Orlando, Florida, USA, 2001-10.

[22] ABEL M-H. ET AL. " Using an Organizational Memory for e-learning", Workshop of Knowledge Management and Organizational Memories, ECAl'2004, Valencia, Spain, 2004- 08-22.

[23] Benayache, A." Construction d'une mémoire organisationnelle de formation et évaluation dans un contexte e-learning". le projet MEMORAe. Thèse de doctorat de l'université de technologie de Compiègne, 2005.

[24] Pascal Lando."Conception et développement d'applications informatiques utilisant des ontologies": application aux EIAH, rjc_eiah, Mai, 2006.

[25] Nathalie Hernandez, Josiane Mothe, Bachelin Ralalason, Patricia tolf, CIDE.9."Modèle de représentation sémantique des documents électroniques pour leur réutilisabilité dans l'apprentissage en ligne". Fribourg, Suisse, 18-20 September 2006.

[26] Nathalie Hernandez, Josiane Mothe, Andriantiana Bertin Olivier Ramamonjisoa, Bachelin Ralalason, Patricia Stolf. "Indexation multi-facettes des ressources pédagogiques pour faciliter leur ré-utilisation", vol. RNTIE-12, pp.207-230, 2008. 